\title{
Cosmovisión Maya reflejada en palabras y conceptos relacionados con desarrollo sostenible, ecología y agroecología
}

\author{
Francisco J. Rosado-May ${ }^{1}$ \\ Hilario Poot Cahun ${ }^{2}$
}

\begin{abstract}
Resumen
La cosmovisión de los Mayas Yucatecos refleja una dimensión ecológica tal y como se muestra a través de palabras y conceptos que guían el manejo de sus sistemas de producción de alimentos. Las actividades de diferentes profesionistas relacionadas con el desarrollo comunitario sostenible, en poblaciones maya-hablantes de la península de Yucatán, requieren del uso de términos ecológicos en su interacción con los pobladores. El presente trabajo hace una exploración de los términos ecológicos más conspicuos en lengua Maya, usados en contextos prácticos por maya-hablantes en sistemas tradicionales como la milpa, y los compara con el uso de esos conceptos en publicaciones académicas relacionadas con el manejo de recursos naturales. Los conceptos ecológicos/agroecológicos analizados son: ecosistema, equilibrio dinámico, nicho, competencia, capacidad de carga, sostenibilidad/resiliencia e investigación acción participativa.

Palabras-Clave: Desarrollo comunitario sostenible; ecosistema: equilibrio dinámico; nicho; competencia; capacidad de carga; sostenibilidad/resiliencia; investigación acción participativa.
\end{abstract}

\section{Abstract \\ Maya World Vision Expressed through Words and Concepts Related to Sustainable Development, Ecology and Agroecology}

\footnotetext{
${ }^{1}$ Indígena maya originario de Felipe Carrillo Puerto, Quintana Roo, México. Ph.D. en Biología por la Universidad de California, Santa Cruz (UCSC), con énfasis en Agreocología. Con más de 30 años de experiencia internacional en investigación y educación superior, sus líneas de trabajo son Agroecología y Educación Intercultural con el objetivo de generar conceptos y métodos relacionados con el desarrollo intercultural, mediante el entendimiento epistemológico del conocimiento indígena. Su experiencia incluye haber colaborado en UCSC, Universidad de Nuevo México, el Colegio del Atlántico en el estado de Maine, Universidad Nacional de Costa Rica, Universidad de Quintana Roo y Universidad Intercultural Maya de Quintana Roo, de la cual fue Rector Fundador. Colega de Hilario Poot Cahun desde 2008. Universidad Intercultural Maya de Quintana Roo. Carretera Muna-Felipe Carrillo Puerto, km137 S/N, Presumida, Quintana Roo, México. francisco.rosadomay@uimqroo.edu.mx

${ }^{2}$ Indígena originario de Tihosuco, Quintana Roo, México, hablante nativo del maya yucateco. Graduado en Lengua y Cultura por la Universidad Intercultural Maya de Quintana Roo. En el verano de 2010 tomó el Seminario "Liderazgo de jóvenes indígenas" en la Universidad de Arizona y en julio 2014 el taller: "Documentación y Revitalización de Lenguas Indígenas" en la Universidad de Texas, Arlington. En otoño de 2014 realizó una estancia de investigación en el Centro de Estudios Mayas de la UNAM. Es profesor de lengua maya y traducción. Ha dirigido tesis de licencitura sobre la didáctica de la traducción y sobre proceso de enseñanza-aprendizaje del maya yucateco. Ha sido estudiante y colega de Francisco J. Rosado-May desde el 2008. Universidad Intercultural Maya de Quintana Roo. Carretera Muna-Felipe Carrillo Puerto, km137 S/N, Presumida, Quintana Roo, México. pootcahunhilario@gmail.com
} 
Yucatec Maya world vision reflects an ecological dimension present in words and concepts that guide their management decisions in their traditional food production systems. Professionals working in different activities related to sustainable community development, require good understanding of agroecological concepts in Maya, especially in their interaction/conversations with local Maya people. This paper explores conspicuous ecological concepts in Maya language used in traditional systems such as the milpa and contrasts them with concepts used in academic papers related to natural resources management. The ecological/agroecological concepts analyzed are ecosystem, dynamic equilibrium, niche, competition, carrying capacity, sustainability/resilience and participatory action research.

Key Words: Community sustainable development, ecosystem, dynamic equilibrium, niche, competition, carrying capacity, sustainability/resilience, participatory action research.

\section{Introducción}

En las comunidades rurales el concepto de desarrollo comunitario sostenible descansa, principalmente, en sus sistemas de producción de alimentos, incluyendo plantas, animales y otros recursos naturales en su entorno. Por tanto, la agroecología ha sido considerada como piedra angular para los procesos de desarrollo comunitario (e.g. Altieri 1990; Atran, 1993; Sevilla Guzmán y Soler Montiel, 2009). Los agroecosistemas sostenibles mantienen los recursos naturales sobre los cuales descansan, hacen uso mínimo de insumos externos al sistema, manejan el impacto de plagas y fitopatógenos a través de mecanismos internos de regulación de sus poblaciones y se caracterizan por su capacidad de recuperación (resiliencia) después de perturbaciones causadas por el proceso de cultivación y cosecha (Gliessman, 2015). Un agroecosistema es un ecosistema compuesto principalmente por los componentes de los cultivos; tiene estructura, poblaciones, comunidades y cada elemento que lo integra tiene una función. La interacción de las funciones de los componentes del sistema, permiten que emerjan propiedades que caracterizan a uno u otro agroecosistema. Lo que emerge de las interacciones explica la sostenibilidad o no de algunos agroecosistemas. De esta manera, los agroecosistemas se pueden caracterizar y entender por la forma en la que se expresa el equilibrio de las poblaciones, de las comunidades o del ecosistema; cada integrante de una población seguramente tiene un nicho que se puede definir; quizá entre las poblaciones habrá algún tipo de competencia por recursos hasta alcanzar o rebasar la capacidad de carga del agroecosistema. Este conjunto de elementos conducen o no hacia la sostenibilidad del agroecosistema, y son manejados de una u otra forma por los milperos (Rosado-May, 2014, 2016), por lo que se deduce que deben haber desarrollado palabras que expresen esos conceptos.

Ahora bien, el proceso de comunicación entre los actores comunitarios y externos que no tienen los mismos elementos culturales y/o lingüísticos locales, interesados en colaborar con la comunidad para su desarrollo sostenible, sería más eficaz si ambas partes tienen el mismo entendimiento conceptual de las bases ecológicas sobre las que se construirá la sostenibilidad. Los conceptos ecológicos son claramente una manifestación de la cultura conocida como occidental, generado y explicado en otro idioma que no es el local ni el indígena. Esta observación es, de hecho, un elemento crucial para el éxito o fracaso de proyectos de desarrollo comunitario, tal y como reconoce la UNESCO (2012) y como han reportado investigadores como Okagor (2014) en el caso de proyectos en el estado de Ebonyi en Nigeria.

Con respecto a la población Maya yucateca, los trabajos relacionados con desarrollo comunitario han sido abordados por diferentes autores. Por ejemplo, Faust (2001) ofrece 
ejemplos de éxitos y fracasos de programas que se han llevado a cabo en la Península de Yucatán relacionados con el cuidado del ambiente. Anderson y Anderson (2011) presentan datos de éxitos y fracasos de programas de desarrollo comunitario en Quintana Roo. Por su parte, Infante Ramírez y Arce Ibarra (2015), en su trabajo sobre la selva Maya en Quintana Roo, identifican terminología Maya para estados vegetacionales como la selva y el acahual. Es interesante notar que ambos trabajos reportan casos principalmente provenientes de comunidades Mayas. Por tanto, se refuerza la noción de que el trabajo de desarrollo comunitario sostenible se ve favorecido cuando existen elementos lingüísticos que permiten comunicar conceptos ecológicos complejos relacionados con la sostenibilidad.

En la revisión de literatura hecha para este trabajo, buscando información que vincule el proceso de desarrollo comunitario sostenible con elementos lingüísticos propios de la cultura Maya, no se encontró antecedente alguno. Asumiendo que la ecología y la agroecología son indispensables para el desarrollo comunitario sostenible, y que ambos tienen elementos en común con los saberes que han desarrollado los pueblos originarios, este trabajo plantea que existen palabras y conceptos Mayas que expresan la esencia de los conceptos ecológicos y agroecológicos aplicables al desarrollo comunitario sostenible, y que, al hacer visibles palabras y conceptos Mayas relacionados con conceptos ecológicos/agroecológicos, se facilita el acercamiento intercultural eficaz entre comunidades Mayas y las personas de diferentes organizaciones interesadas en el trabajo que conduce al desarrollo comunitario sostenible.

\section{Metodología}

En la literatura sobre milpa es posible encontrar términos ecológicos expresados de diferente manera. Como ejemplo están los trabajos de Hernández Xolocotzi y Padilla Ortega (1980); Teran y Rasmussen (1994); Hernández Xolocotzi et al. (1995); Rosado-May (2012) y Mariaca Méndez (2015). La revisión de esos trabajos, aunado a visitas de campo interactuando con milperos en Quintana Roo, Yucatán y Tabasco, así como la experiencia personal de los autores como descendientes de milperos, permitió la elaboración de una lista de los términos (Rosado-May 2014) más conspicuos relacionados con ecología/agroecología. Esta lista incluye: ecosistema, equilibrio dinámico, nicho, competencia, coexistencia, capacidad de carga, sostenibilidad/resiliencia e investigación acción participativa.

Es importante mencionar que aun cuando se hizo una revisión de literatura y diccionarios para respaldar la escritura de las palabras en Maya, los autores decidimos escribirlas con base en la convención vigente. Por ejemplo, actualmente se sustituye la letra " $h$ " por " $j$ " o la " $z$ " por "s", para emitir un sonido acorde con el español.

A continuación, se presenta la descripción de cada concepto con base en información científica. Para cada concepto se presenta una expresión en Maya, español, coloquial y de cuyo contexto se puede apreciar mejor la aplicación del concepto en una conversación de tipo cotidiano.

\section{Resultados}

\section{Ecosistema/Yóok'ol kaab}

La palabra ecosistema se atribuye a Tansley (1935). De acuerdo con Odum (1953), cada ecosistema tiene componentes (estructura), y cada uno de esos componentes tiene una función en el ecosistema. La interacción de los componentes permite la emergencia de propiedades del ecosistema, tales como ciclo del agua, ciclo de nutrientes/biogeoquímicos (movimiento de materiales), flujo de energía, dinámica de poblaciones mediante 
interacciones entre especies que conducen a la regulación de las poblaciones y a las cadenas tróficas.

En el caso de Maya, la palabra yóok'ol kaab es la que mejor expresa la idea de ecosistema. Yóok'ol kaab puede ser interpretado como mundo o como universo. En el universo, de acuerdo con la cosmogonía Maya, se puede encontrar el mundo, el supramundo y el inframundo. Con esta cosmovisión, el concepto de yóok'ol kaab incluso rebasa al concepto de ecosistema, toda vez que el tamaño del ecosistema puede ser delimitado arbitrariamente. La cosmovisión Maya explica las interacciones entre ecosistemas y también que el planeta tierra no está aislado del resto del universo; esta visión ofrece un elemento filosófico y conceptual sólido para entender las interacciones entre especies y la interconectividad entre ecosistemas. Esto explica por qué, entre los Mayas, prevalece la idea de la conservación de los recursos naturales (Infante Ramírez y Arce Ibarra, 2015; ReyesGarcía, 2009; Tuz Chí, 2009).

Ejemplo en contexto. Enseguida, se presenta una conversación cotidiana entre un aprendiz de milpero, el nieto, con un experto, el abuelo. Esta conversación sucede durante la actividad o durante el descanso; es parte de un proceso de enseñanza-aprendizaje para la construcción de conocimiento.

Maya:

A: Nool, ba'axten ka míistik u báak'pach a kool. Ba'ax yaan ti' ka púuts'uk le k'áak'o'.

B: Paal wa ka púuts'uk le k'áak'o' yaan u yeelsik uláak' kúuchilo'ob ma' in ti'ali', k'a'ajse' k'a'abéet ik kanáantik yóok'ol kaab.

\section{Español:}

A: Abuelo, ¿por qué haces guardarraya? ¿Qué importa que se pase el fuego del otro lado?

B: Muchacho, si el fuego pasa del otro lado quemará un área que no me corresponde y puede destruir algo, además debemos cuidar nuestro entorno.

\section{Equilibrio dinámico/Ki' tsool.}

El proceso de equilibrio dinámico se presenta en la interacción de las poblaciones de los diversos organismos presentes en el ecosistema. Una población está formada por individuos de la misma especie. Dentro de la misma especie también puede haber diferentes poblaciones. Un conjunto de poblaciones conforma una o varias comunidades (Odum, 1953). Las poblaciones tienen mecanismos de equilibrio que controlan su tamaño, es decir, no crecen indefinidamente. A través de las interacciones entre los individuos de la misma población (intrapoblacional), o entre individuos de diferentes poblaciones (interpoblacional), se llevan a cabo mecanismos como depredación, asociación, simbiosis, etc., mismos que explican el tamaño de la población (Odum, 1953). La interacción de organismos se refleja en el ecosistema, de modo que va teniendo cambios a través del tiempo, los cuales se observan claramente en etapas/momentos de desarrollo de la vegetación. Por un tiempo se consideró que un ecosistema obtiene la madurez cuando alcanza un clímax, es decir, un nivel de estabilidad (Clements, 1905). La idea de un ecosistema estable fue desechada por Tansley (1935), quien, con base en investigaciones sólidas, introdujo el término de "equilibrio dinámico"; es decir, el ecosistema alcanza un nivel de madurez, pero no es estable, sino que tiene fluctuaciones propias de los procesos de interacción de las poblaciones, lo cual implica una dinámica de poblaciones que se puede graficar dentro de un rango que expresa la madurez del ecosistema. 
En el idioma Maya, existen dos palabras que expresan la idea de equilibrio dinámico, ambas se relacionan entre sí. Por un lado, está la palabra $K i^{\prime}$ tsool, que significa buena adaptación, y por otra está la palabra toj ets'tal, que significa balance (Barrera Vázquez, 1980:801). El primer caso se compone de dos palabras: $K i^{\prime}$ que indica favorable, bueno, adecuado, y tsool que significa estado, adecuado, adaptado. La combinación de ambos es lo que implica un proceso dinámico, no estable. La otra palabra toj ets'tal se compone de dos partes. Toj significa recto, verdad; ets'tal significa equilibrio, balance; no implica ni estable ni inerte ni inmóvil. Así, la combinación de ambas palabras, recto y balance, prácticamente se refiere a un proceso que alcanza un equilibrio dinámico.

Las palabras para expresar comunidad de vegetación tropical de la Península de Yucatán, las ha descrito Taube (2003) con base en el análisis de términos que expresa Barrera Vázquez (1980:150, 454-55) en el Diccionario Maya de Cordemex. Al referirse a las arvenses (plantas herbáceas y arbustos) que crecen en la milpa junto con los cultivos, los Mayas las Ilaman lob'chahal; para denominar vegetación cerrada en una selva, se usa la palabra lob' $k^{\prime} a x$. En ambos casos, la palabra lob implica oscurecimiento, es decir, expresan una característica de crecimiento de la vegetación que es la de crear sombra al suelo y a los organismos debajo de las hojas. Taube cita a Hanks (1990), quien hace una revisión de la interpretación de función del milpero; cuando éste camina por la selva, está debajo de los árboles para cortar algunos elementos del interior de la selva, esto se expresa como yanal $k^{\prime} a x$. Lo anterior es importante porque para la conceptualización Maya del papel del milpero, la persona está debajo de la selva, no está "dentro" o "en" la selva. En otras palabras, en Maya se aprecia una combinación de expresión de comunidad de vegetación y el nicho del milpero.

Ejemplo en contexto. En la vida comunitaria tradicional se lleva a cabo una actividad tan cotidiana cuya importancia pasa desapercibida. Es el momento en el que los mayores se reúnen con los niños y los muchachos. Es el momento de conversar, de reflexionar, de filosofar, de escuchar a los mayores sobre diferentes temas de la comunidad, o para escuchar historias, anécdotas o leyendas que siempre dejan enseñanza. En uno de esos momentos la conversación se desarrolló de la siguiente forma:

Maya:

A: Yuum, jach ch'ench'enkil in wilik tuláakal máax kex yaan ba'alob ku bin u yúuchul ichil le kajtalila'. Je'el bix le máako'ob ku suuto'ob tu meyajo'obo' tak Cancuno', u juum le traktoro'obo', bey mixmáak ilike'.

B: Máasima', yaan jump'éel ki' tsool waye'. Ba'ale' ba'ax ba'alobil ku bin u yúuchul ka wa'aliko'.

A: Je'elbix u yaal le nojoch máak te' ti'its'o tso'ok u k'amikubaj te' noj najil xooko'. Kex leti' le yáax máax náajalt le ba'ax je'el te' kajtalila' bey mixmáak meentik u kuentaile'.

Español:

A: Papá, no entiendo qué pasa. A pesar de que hay movimientos y cambios recientes, como la llegada de los trabajadores de Cancún, la maquiladora trabajando, veo muy tranquilo el pueblo. Parece que no pasa nada.

B: Tienes razón hijo, parece que no hay cambios, que todo sigue igual. ¿Qué llamó tu atención? Ha habido cambios antes y no lo notaste.

A: Por ejemplo, el hijo del señor de la esquina culminó sus estudios universitarios, tiene una carrera. A pesar de ser el primero en la comunidad en ser licenciado, al parecer nadie lo nota. 


\section{Nicho/U Meyaj, U Na'atik.}

El concepto de nicho fue presentado por Grinnel (1908) como un referente de espacio geográfico donde se encuentran condiciones adecuadas para la vida y el desarrollo de alguna especie. Más adelante, Elton (1927) consideró que nicho es el estatus, lo que "hace" el individuo o especie en la comunidad; de aquí se desprende la idea de que el nicho es el papel que realiza el individuo en la comunidad o en el ecosistema. Las definiciones anteriores presentaban algunas dificultades para medir el concepto en la naturaleza, de modo que Hutchinson (1957) presentó la definición de nicho como el hiperespacio que resulta de la interacción y medición de " $n$ " dimensiones conformadas por los componentes biogeoquímicos y climáticos que explican la presencia y el desempeño de alguna especie en un sitio geográfico.

Desde el punto de vista de la lengua y cultura Maya de la Península de Yucatán, se cuenta con palabras que expresan la idea de nicho como el papel que juega el individuo en su comunidad, sea animal, vegetal o humano. No se ha encontrado evidencia de alguna palabra en Maya y su significado conceptual, que implique la percepción matemática de nicho que propone Hutchinson (1957). Para la lengua Maya, nicho es la combinación, en uso conjunto o separado, dependiendo del contexto, de dos palabras: U'meyaj y $U$ na'atik. El desglose de u'meyaj es muy semejante a "el trabajo de alguien", "lo que hace alguien". Esta interpretación se aplica tanto a seres humanos, otras especies de animales y también a plantas y objetos inermes. Cada cosa tiene una función. El desglose de u na'atik es muy semejante a "la forma en que alguien entiende algo", o "el cómo se entiende algo". Esta interpretación básicamente se aplica a seres humanos al explicar su función dentro de la comunidad.

Ejemplo en contexto. La siguiente conversación cotidiana sucede entre la hija y el padre, quien es reconocido en la comunidad como una persona sabia. La muchacha es estudiante de secundaria y aprovecha los momentos en los que su padre y su madre se sientan a platicar sobre cosas de la vida, de la escuela, de la comunidad, de política, etc.

Maya:

A: Yuum, a'ala'ab teen te' najil xooko' tuláakalo'on bine' yaan jump'éel ba'ax ik biilal way yóok'ol kaabe'. Tu ya'alaj ten le ajka'ansajo' ich káastelan bine' Nicho. Bix u páajtal in wa'alil le bey ich maayao'.

B: A meyaj. Ba'ax taaja'anech a meent way yóok'ol kaabe' a meyaj. Tuláakalo'one' yaan jump'éel ba'ax k meentik te' lu'uma', le je'elo' ik meyaj.

Español:

A: Papá, aprovechando esta plática, me dijeron en la escuela que todo ser viviente, como nosotros, tenemos una razón de existir, que cada uno es importante dentro de la comunidad y que cada uno tiene un trabajo. Me dijeron que esto es conocido como nicho. ¿Cómo lo puedo decir en maya?

B: Hija, cada uno tenemos una labor diferente dentro de la comunidad. Aunque parece que varias personas que hacen la misma labor son iguales, en realidad, son diferentes. Tu trabajo y cómo lo haces te distingue de los demás, y por eso eres importante para la comunidad. Todos tenemos algo con qué contribuir en este mundo, eso es nuestro trabajo.

Competencia/No existe equivalencia conceptual en Maya; por tanto, no existe palabra que tenga ese significado. 
El término competencia ha sido muy usado en el campo de la ecología. Se estima que su origen está en el trabajo de Forbes $(1880,1887)$, estudio en el que analizó las interacciones de organismos en ecosistemas acuáticos. El concepto de competencia es comúnmente utilizado en agronomía para hacer referencia al buen desarrollo de los cultivos, en los cuales, se supone, que los organismos compiten entre sí para obtener recursos que son limitados, tales como luz, el agua, los nutrientes, el espacio y otros factores. Se asume, también, que el organismo que mejor compite es el que sobrevive.

El concepto de competencia no se ha detectado en los saberes o conductas de los pueblos originarios que aún conservan sus tradiciones propias. Esto explica la falta de palabras que expresen ese concepto. Así lo demuestra el trabajo de Rogoff (2011) con los Mayas de San Pedro en Guatemala, o el trabajo de Bolin (2006) en comunidades Incas de las montañas de Perú, especialmente en Chillihuani, o las descripciones que hace Thomas (1858) de los Cherokees, o de Gaskins (1999) con los Mayas de Yucatán. La palabra competencia no tiene un equivalente en Maya; sólo podría existir si alguien acuña un neologismo, pero sin tener el respaldo cultural tradicional.

Ejemplo en contexto. La siguiente conversación se ha repetido, palabras más, palabras menos, en cada ocasión semejante, sea en un aniversario de una escuela, de un cumpleaños o de alguna celebración que implique la aportación de los participantes.

Maya:

A: Na', sáamale' yaan ik bisik jejeláas ba'alo'ob uti'al ik k'iimbesik úuchik u káajal u meyaj le najil xooko'. Ba'ax ken in bisej.

B: Mixba'aj ken a bis ko'oj. Mina'an to'on, ku ts'o'okole' ma' suuk ik meentik le ba'ax beyo' tumen ku taasik ba'ateil. Ma'atech wáaj a wilik bix ik bisik u nu'ukulil ken meenta'ak Waajil Kool. Tuláakalo'on kik bisik le ba'ax je'el ik ts'a'ayalo'. Ma'atech ik ilik máax j-bis ba'ax asab ko'oj. Ma'atech ik ketlam ti' le ba'axo'ob beyo'.

Español:

A: Mamá, nos pidieron que mañana llevemos aportaciones para conmemorar el aniversario de la escuela. ¿Qué podría llevar?

B: No llevarás algo caro, hija. Recuerda que no tenemos dinero. Además, se trata de colaborar, no de llevar cosas caras porque esas cosas generan envidias y disgustos. ¿No te das cuenta cómo aportamos para la primicia? Todos llevamos lo que podemos. Nunca nos fijamos quién llevó más o quién llevó menos.

\section{Coexistencia/Toj ets'tal, Toj óolal.}

La idea de coexistencia parte del modelo de competencia de Lotka-Volterra, el cual predice que si las especies en competencia regulan su propio crecimiento a través de mecanismos intraespecíficos (Lotka, 1920; Volterra, 1931), entonces coexisten. Si bien el concepto de coexistencia nace del concepto de competencia (el cual se mantiene en esencia en las diferentes manifestaciones conceptuales de coexistencia), permite la apertura de un espacio de entendimiento diferente de los procesos que explican la diversidad y abundancia de especies en áreas reducidas como en los trópicos.

En el saber Maya no hay espacio para el concepto de competencia, la explicación a la presencia de las especies en una comunidad se explica por la coexistencia. Es decir, la interacción de especies permite los avances de adaptación a su entorno cambiante, 
precisamente, por su capacidad de coexistir, no de competir. Esta percepción se observa incluso en el tejido social comunitario indígena, e incluso se expresa en sus interacciones comerciales (Rosado-May y Cuevas Albarrán 2015), en las cuales prevalece la coexistencia como una forma de mantener la comunidad funcionando.

Las palabras Mayas que reflejan un concepto muy parecido al de coexistencia, son Toj ets'tal y Toj óolal. La palabra Toj significa recto o verdadero; la palabra ets'tal significa condición, equilibrio o balance. La palabra óolal significa calma o consolación. Ambas palabras, dependiendo del contexto, pueden implicar el equivalente a coexistencia, y normalmente, pero no únicamente, se refieren a conductas de seres humanos. Es decir, la coexistencia sucede cuando los participantes humanos son rectos, sinceros y verdaderos en su actuación entre sí; esta actitud conlleva a que la comunidad tenga calma, esté en paz. Las investigaciones de autores mencionados en el apartado de competencia, Rogoff (2011), Bolin (2006), Thomas (1858) y Gaskins (1999) respaldan la afirmación anterior; esto es, las comunidades indígenas tradicionales crean condiciones de coexistencia comunitaria como una forma de avance en su desarrollo $\mathrm{y}$, culturalmente, han creado condiciones que promueven la coexistencia.

Ejemplo en contexto. Esta conversación es una continuación de la que se inició con el concepto anterior.

Maya:

A: Wa taláakale'ex ka bisike'ex a wáantaje'ex ti' le k'iimbesaj keeto' asab ma'alob, tumen beyo' mixtan a wilik máax asab nojoch wa chichan ba'a tu bisaj. Beytuno' ku yaantal jump'éel toj ets'tal ichilaba'ex.

B: Ts'o'ok in na'atik na'. Jaaj bey in wilik u yaantal toj óolal ichil le máaxo'ob ku táakpajalo'ob ti' le waajil koolo'.

Español:

A: Hija, si todos llevaran su colaboración para el aniversario, sin que haya eso de ver quien lleva lo más caro o lo más grande, entonces sería mejor, porque de esa manera no habría preocupación ni envidia. Entonces existiría más armonía entre nosotros.

B: Ya entendí mamá. Es lo mismo que observo con los participantes de la primicia, todos mantienen una armonía, un equilibrio.

\section{Capacidad de carga/Kanan K'áax, Yo'o bej.}

El concepto de capacidad de carga no tiene una génesis tan clara como los anteriores. Es un término muy socorrido, especialmente en materia de política ambiental, pero a la vez confuso. Los diccionarios lo definen como el "máximo número de organismos que una superficie puede soportar". La evidencia más antigua del uso del término se remonta al año de 1845, cuando el Secretario de Estado de los Estados Unidos de América presentó una iniciativa para regular el comercio exterior (Sayre, 2008). Al parecer, el origen del término no es resultado de una investigación ecológica. Gabb (1873) parece ser el primero en reportar el comportamiento de poblaciones de animales silvestres en Santo Domingo. Leopold (1933) estudió la dinámica poblacional de varias especies silvestres, considerando la disponibilidad de alimentos como base para una estrategia de manejo. Odum (1953) adopta el término, pero expande la noción del concepto al presentar la idea de que las poblaciones silvestres pueden alcanzar un equilibrio dinámico como una forma de expresión de la capacidad de carga, debido a los atributos de un ecosistema dinámico. 
El concepto de capacidad de carga ha recibido numerosas críticas, especialmente cuando se usa para fines malthusianos. En materia de planeación turística, cuando se trata de determinar el número de personas adecuado para un lugar determinado, sea en forma de hotel o en una reserva natural, el concepto de capacidad de carga no ha funcionado; en su lugar se aplican metodologías que descansan en una combinación de medición de factores ecológicos y un proceso de consenso entre los diversos actores involucrados en la zona sujeta a planificación y política ambiental (Rosado-May \& Kissmann, 1998).

Una vez entendido el sentido del concepto de capacidad de carga, al revisar la lengua Maya se encuentran dos palabras que pueden ser tomadas como elementos para establecer comunicación eficaz. Las palabras asumen que el concepto de capacidad de carga es solamente un referente, no una fórmula mágica que determina una población estática. En este sentido, las palabras no se traducen directamente como equivalentes a capacidad de carga, sino como palabras que indican el camino para consensar una determinada población o uso de un recurso natural. Las palabras son: Kanan K'áax y Yo'o bej. Dependiendo del contexto, se pueden usar juntas o separadas. La palabra kanan significa cuidar, amparar, guardar, proteger, vigilar. La palabra k'áax quiere decir selva, montaña, campo, desmonte, deslindar, flora, llover, naturaleza. Por otro lado, la palabra Yo'o implica "estar arriba o encima de", "ambiente"; mientras que la palabra bej quiere decir camino, acceso, actual, brecha, sendero, trayecto, vía. Al unir las palabras Kanan K'áax, prácticamente se está tratando de decir que se debe tener cuidado con la naturaleza, se refiere al manejo de los recursos naturales; no sería muy atrevido señalar que ambas palabras tienen un contexto de conservación y de sostenibilidad (Burford et al., 2012). Asimismo, las palabras Yo'o bej quieren decir que "algo o alguien" está "en el camino correcto, en la dirección adecuada"; tampoco sería muy arriesgado decir que ambas palabras implican que "el ambiente está en la vía correcta". Bajo este análisis, no queda duda de que ambas palabras, Kanan K'áax y Yo'o bej, representan un contexto de manejo adecuado de los recursos naturales, del ambiente, para que la naturaleza esté en el camino correcto. Es decir, representan una expresión de capacidad de carga sostenible en un sistema dinámico en el que hay participación de personas.

Ejemplo en contexto. El caso del aprendiz, muchacho en edad de aprender a hacer milpa, y de su abuelo, quien es experto. El aprendiz quiere saber por qué hay que preparar el terreno en lugares diferentes cada determinado número de años. Se refiere a desmontar, tumbar.

Maya:

A: Ba'axten mixtan a ka'a kolik le k'áaxo' nool

B: Tumen ts'o'ok u ti'ibital in máatan waye', ka'abéet in p'atik wa jayp'éel ja'ab uti'al u ka'a ch'a'ik u yóolal le lu'umo'. Beyo' táan ik kanáantik le k'aaxo'.

A: U k'áat u ya'ale' yo'o beej yanech.

B: Jach beyo'

\section{Español:}

A: ¿Por qué no tumba monte de nuevo en el mismo sitio, abuelo?

B: Porque el suelo, en esta parte de monte, ha llegado a su límite. Necesitamos dejarlo que descanse solito por unos cuantos años para que se recupere. De esta forma, estamos cuidado nuestros propios recursos.

A: Entonces, eso significa que está haciendo lo correcto.

B: Así es. 


\section{Sostenibilidad/Resiliencia/Ma'alob Kuxtal, Kanan K'áax y Yo'o Bej}

La palabra sostenible, usada aquí como sinónimo de sustentable, tuvo mucha notoriedad a partir del informe Brundtland (1987), documento en el que el desarrollo sostenible es entendido como aquel que puede satisfacer las necesidades de las generaciones presentes, sin comprometer las posibilidades de desarrollo de las generaciones futuras para atender sus propias necesidades. El concepto ha guiado políticas públicas e inspirado numerosas investigaciones.

Capacidad de carga, resiliencia y sostenibilidad están estrechamente relacionadas. El concepto de resiliencia fue introducido por Holling (1973) para tratar de entender la dinámica de los ecosistemas, y se define como la capacidad de un sistema para resistir perturbaciones sin cambiar en forma significativa sus propios procesos y estructura (también se define como estado estable alternativo).

Con base en la idea expresada en el párrafo anterior, en la lengua Maya se encuentran las palabras Ma'alob Kuxtal, Kanan K'áax y Yo'o Bej. Las dos últimas, Kanan K'áax y Yo'o $B e j$, se han analizado en la discusión sobre capacidad de carga; este análisis es también válido para efectos del concepto de sostenibilidad. La otra palabra, Ma'alob Kuxtal, tiene el siguiente significado: Ma'alob quiere decir bueno, apto, arreglo, desarrollar, error, firmeza, disfrutar, imperfecto, revisar, mejorar; mientras que kuxtal hace alusión a bienestar, convivencia, existencia, revivir/revitalizar, subsistir, resiliencia, vida, vivir. La combinación de ambas palabras, Ma'alob kuxtal, podría traducirse como "buen vivir", "resiliencia" y "capacidad para mantenerse bien"; es decir, básicamente se está refiriendo a la sostenibilidad. La discusión del significado de estas palabras en Maya, desde el punto de vista epistemológico, indica que, conceptualmente, los Mayas tenían muy clara la interrelación de los elementos de la naturaleza, incluyendo el ser humano. En el lenguaje occidental (sea inglés, español u otra lengua) la mejor interpretación sería sostenibilidad o resiliencia, dependiendo del contexto. Las tres palabras pueden usarse por separado o juntas, depende del contexto de la conversación.

Ejemplo en contexto. La siguiente conversación indica que en el pensamiento Maya la idea asociada a la sostenibilidad está siempre presente, no a nivel de preocupación, sino de ocupación y acción, el vivir bien, cubriendo adecuadamente las necesidades básicas de la familia.

Maya:

A: Nool, bix je'el u yaantal to'on jump'éel ma'alob kuxtale'.

B: Paal jump'éel ma'alob kuxtale' ma' u k'áat u ya'al ka yanak tech ya'abkach taak'ini'. Ma'alob kuxtale' u k'áat u ya'ale' ka yanak to'on u ti'ibilil ti'al u bin to'on tu beel, je'elbix toj óolal, jaanal yéetel otoch. Beyxan ka ik bis jump'éel ma'alob bisbail yéetel le yóok'ol kaaba'. Ka'abéet ik kanáantik tumen ti' leti' ku taal tuláakal ba'ax yaan to'on.

Español:

A: Abuelo, sé que somos pobres, pero también sé que los mayores procuran que estemos bien. ¿Cómo es que podemos vivir bien?

B: Hijo, vivir bien no significa tener mucho dinero. Vivir bien es tener lo suficiente para estar en armonía entre nosotros, tener salud, alimentos y hogar. De igual manera, debemos saber cómo cuidar lo que nos da de comer, hacer buen uso de los recursos naturales, debemos cuidarlos porque de ahí proviene todo lo que tenemos. 


\section{Investigación acción participativa/Tsikbal.}

Una de las metodologías de trabajo para Agroecología, tanto en investigación como en desarrollo comunitario, es la investigación-acción participativa (IAP) (Méndez et al., 2015). El concepto de IAP ha tenido cambios a través de tiempo. En 1994, Chambers dió a conocer la metodología de trabajo conocida como "Participatory Rural Appraisal (PRA)", un antecedente de IAP. El mismo autor, en 2008, consideró que IAP no es un cuerpo monolítico de ideas y métodos, sino una orientación plural a la forma de construcción de conocimiento y cambio social. En un trabajo más reciente, Chevalier y Buckles (2013) identificaron la parte de Participación de IAP como la vida en la sociedad; la parte de Acción como la experiencia, y la parte de Investigación como el conocimiento. La interacción de estos tres elementos da origen a IAP.

Con base en la discusión del párrafo anterior, podemos señalar a la interculturalidad como elemento importante en la investigación participativa (Rosado-May, 2015), lo cual no es una apreciación reciente. El término intercultural lo es, pero el proceso no lo es. En 1995, Fals Borda, sociólogo colombiano, ofreció una conferencia magistral en Atlanta, en la reunión de la Sociedad Sureña de Sociología, con un tema directamente relacionado con IAP. Ahí, Fals Borda demandó a los científicos que no monopolicen ni impongan en forma arrogante su conocimiento, sino que, con respeto, compartan sus habilidades y conocimientos con aquellos de las comunidades en donde llevan a cabo sus investigaciones, de modo que ambos actores, científico y personas de la comunidad, sean coautores del conocimiento que emerge de esa interacción (Fals Borda, 1995). Fals Borda es reconocido como uno de los pioneros de IAP en el mundo (Hall, 2005; Ortiz y Borjas, 2008).

Desde el punto de vista de la construcción de conocimiento, la investigación es sólo un componente de un proceso más complejo. Para hacer una buena investigación se requiere haber pasado por un proceso de aprendizaje. Esta afirmación es válida tanto para los sistemas indígenas como para los no indígenas.

Cualquier persona inmersa en un proceso de aprendizaje o haciendo investigación o trabajo comunitario, necesariamente hace uso de la comunicación en formas diversas. Para los Mayas, este proceso está mediado por un sistema que tiene como vehículo las palabras, pero que las palabras no necesariamente son lo más importante en el sistema (Rosado-May, 2016); el proceso de acción participativa, sea para aprender o para investigar, es una excelente forma en la que existe transferencia de saberes, valores, conocimientos, prácticas, diversas actividades y acciones que, eventualmente, conforman el complejo cultural que permite la creación de nuevos saberes, conocimientos, prácticas, actividades, acciones y demás (Rosado-May, 2016). Esta parte es especialmente importante de mencionar y de enfatizar.

Habiendo explicado lo anterior, ¿qué palabra o palabras en Maya reflejan el proceso que se explicó en el párrafo anterior? La respuesta la contiene la palabra tsikbal, la cual ha llamado la atención de muchos investigadores. Los diccionarios maya-español en línea: aulex.org/es-myn/ y www.mayas.uady. mx/diccionario/ indican como traducción de tsikbal al español las siguientes palabras: charla, conferencia, conversación o conversar, cuento o contar, departir, dialogar, historia, narrar, platicar, relatar, rumor y secreto. Entre los Mayas, esas palabras forman parte de un proceso mucho mayor que una simple conversación. Tsikbal refleja un proceso de transmisión y construcción de conocimiento que es básico para la vida y el desarrollo de la comunidad; es un concepto que combina palabra con lenguaje corporal, acción manual y práctica constante (Rosado-May, 2016). 
Con base en lo anterior, la mejor forma de interpretar al tsikbal es a través de la metodología de aprendizaje/investigación con acción participativa. En el contexto de esta interpretación, la palabra investigación se refiere a una forma de búsqueda de conocimiento, de saberes; es decir, la investigación no es una actividad solamente académica. El tsikbal es la parte operativa, visible y no tangible, de un proceso comunitario que, a través de la generación eficaz de saberes, conocimientos, acciones, conduce su desarrollo. El proceso de comunicación que ofrece el tsikbal no solamente es oral, también incluye comunicación no verbal, que facilita el desarrollo de la observación holística, habilidad clave para el aprendizaje e innovación del conocimiento.

Ejemplo en contexto. La siguiente conversación sucede a menudo entre personas de la comunidad y visitantes, especialmente aquellos con interés en aprender a través de investigación, sea académica o periodística.

Maya:

A: Nojoch maak, taalen tsikbal ta wéetel.

B: Ba'ax taak a tsikbaltik.

A: Yosal le k'immbesaj ka meentike'ex waye'. Jach uts tin wich. K'as taak in wojeltik bix u tsoolil u meenta'al.

B: Ma'alob, bix ma'. Páaybe'en ik ts'áik k'ajóoltbil ik suukbenilo'ob. Ko'ox, ch'eenxikint ba'ax ken in tsikbalt ikil ik táakpajal ichil le k'iimbesaja'.

Español:

A: Disculpe señor, me gustaría platicar con usted.

$B$ : ¿Sobre qué quiere hablar?

A: Veo que hacen esta celebración en este pueblo. Me parece interesante. Me gustaría saber un poco sobre ello. ¿Cómo se organizan para llevarlo a cabo?

B: Bien, con mucho gusto. Es importante dar a conocer nuestras tradiciones. Venga conmigo, escúcheme y participemos en la celebración.

\section{Discusión}

La cultura Maya es reconocida, entre otras cosas, por el manejo sostenible de sus recursos naturales. Una explicación es que su cosmovisión jugó un papel importante en los procesos que condujeron a esos niveles de sostenibilidad. Esa cosmovisión aún está presente, pero no se está transmitiendo a las nuevas generaciones con la misma fuerza que hace unos años. Aún se puede observar su aplicación en el trabajo cotidiano en los campos de cultivo y en la selva, así como en el lenguaje que usan en la vida cotidiana, pero no es fácil su detección.

Asumiendo que, desde el punto de vista científico, la Ecología nos permite entender los procesos que explican la sostenibilidad de sistemas naturales, y la agroecología hace lo propio en sistemas de producción de alimentos, el reto fue encontrar los momentos y expresiones que reflejen conceptos más que traducciones o interpretaciones de las palabras. Con esta base y con el tsikbal se pudieron encontrar significados epistemológicos de palabras actuales del Maya de Yucatán que se acercan a los conceptos de ecología y agroecología.

Entender el significado epistemológico de palabras Mayas de la Península de Yucatán, que expresan conceptos ecológicos sofisticados, permite construir un puente sólido de intercambio de saberes entre Mayas y no Mayas, quienes tienen diferentes formas de 
aprendizaje, construcción e innovación de conocimiento. Este puente es necesario para temas de asesoría, extensionismo, difusión y, especialmente, co-construcción de conocimiento científico. Los análisis presentados en los párrafos que discuten el significado equivalente entre conceptos complejos de origen occidental y ecológicos, indican que los Mayas de la Península de Yucatán también fueron capaces de generar un léxico que registra palabras que expresan conceptos complejos y sofisticados. En realidad, no hay otra forma de entender el gran avance que tuvo esta cultura prehispánica. Para lograr alcanzar una visión del mundo como la que los investigadores más renombrados expresan de los Mayas, para alcanzar la sofisticación en su ingeniería, matemática, entendimiento y manejo de sus recursos naturales, de su agricultura, o incluso del arte de la guerra y de construcción de consensos, necesariamente debieron tener un sistema de comunicación altamente sofisticado.

La investigación epistemológica de la lengua local también tiene otra ventaja: empodera a los maya-hablantes, en este caso, porque les permite interactuar con el "otro" de la cultura occidental en un nivel de alta sofisticación con respecto a saberes y construcción de conocimiento local. Esto genera un clima social que propicia condiciones adecuadas para rehacer el tejido social comunitario para alcanzar el desarrollo sostenible. Cualquier otro camino, como señalan Anderson \& Anderson (2011) y Faust (2001), lleva a situaciones que evitan el desarrollo local al ritmo que la sociedad necesita.

El tsikbal está abierto.

\section{Referencias}

ANDERSON, Eugene N. \& ANDERSON, Barbara. Development and the Yucatec Maya in Quintana Roo: some successes and failures. Journal of Political Ecology, v.18, p.51-65, 2011.

ALTIERI, Miguel A. Agroecology and rural development in Latin America. In: Altieri, M.A. and S.B. Hecht (Eds.) Agroecology and small farm development. Boca Raton: CRC Press Inc., 1990.

ATRAN, Scott. Itza Maya tropical agro-forestry. Current Antropology, v. 34, n. 5, p. 633700, 1993.

BARRERA VÁSQUEZ, Alfredo. (Ed.). Diccionario Maya Cordemex: Maya-Español, EspañolMaya. Mérida, Yucatán, México: Ediciones Cordemex, 1980.

BOLIN, Inge. Growing up in a culture of respect. Child rearing in Highland Peru. Austin: University of Texas Press, 2006.

BRUNDTLAND, Gro H. 1987. Report of the world commission on environment and development: Our common future. UN Documents. http://www.un-documents.net/ourcommon-future.pdf, consultado el 27 de diciembre de 2019. 1987

BURFORD, Gemma; KISSMANN, Susanne; ROSADO-MAY Francisco J.; ALVARADO DZUL, Santos H. \& HARDER, Marie K. Indigenous participation in intercultural education: learning from Mexico and Tanzania. Ecology and Society, v. 17, n. 4, p. 33, 2012.

CHAMBERS, Robert. Participatory Rural Appraisal (PRA): Analysis of Experience. World Development, v. 22, n. 9, p. 1253-1268, 1994.

CHAMBERS, Robert. PRA, PLA and Pluralism: Practice and Theory. In P. Reason and $\mathrm{H}$. Bradbury (eds.), The Sage Handbook of Action Research: Participative Inquiry and Practice. 2nd edition, pp. 297-318. London: Sage, 2008.

CHEVALIER, Jacques M. \& BUCKLES Daniel J. Participatory Action Research: Theory and Methods for Engaged Inquiry. New York: Routledge, 2013.

CLEMENTS, Frederick E. Research methods in Ecology. Lincoln: University Publishing Company. Reprinted New York: Arno Press (1977). 1905. 
ELTON, Charles S. Animal ecology. London: Sidgwick and Jackson, 1927.

FALS BORDA, Orlando. Research for social justice: Some North-South convergences. Plenary Address at the Southern Sociological Society Meeting, Atlanta, April ${ }^{\text {th }}, 1995$.

FAUST, Betty B. Maya environmental successes and failures in the Yucatan Peninsula. Environmental Science and Policy, v. 4 n. 4-5, p. 153-169, 2001.

FORBES, Stephen A. On some interactions of organisms. Bulletin Illinois State Laboratory of Natural History, v. 1, p. 3-17, 1880.

FORBES, Stephen A. The lake as a microcosm. Bulletin Science Association of Peoria, Illinois, v. 1887, p. 77-87, 1887.

GABB, William M. On the topography and geology of Santo Domingo. Transactions of the American Phylosophical Society, v. 15, n. 1, p. 127, 1873.

GASKINS, Suzanne. Children's daily lives in a Mayan Village: A case study of culturally constructed roles and activities. In A. Göncü (Ed.), Children's engagement in the world, (pp. 25-61). Cambridge: Cambridge University Press, 1999.

GLIESSMAN, Stephen R. Agroecology: The Ecology of Sustainable Food Systems, 3rd ed. Boca Raton, FL: CRC Press/ Taylor \& Francis, 2015.

GRINNEL, Joseph. The biota of San Bernardino Mountains. University of California Publications in Zoology, v. 5, p. 1-170, 1908.

HALL, Budd L. In from the Cold: Reflections on Participatory Action Research From 19702005, Convergence, v. 38, n. 1, p. 5-24, 2005.

HANKS, William F. Referential practice: language and lived space among the Maya. Chicago: University of Chicago Press, 1990.

HERNÁNDEZ XOLOCOTZI, Efraim; BELLO SALAZAR, Eduardo y LEVY TACHER, Samuel. La Milpa en Yucatán. Un sistema de producción agrícola tradicional. Tomo 2. Texcoco, México: Colegio de Posgraduados, 1995.

HERNÁNDEZ XOLOCOTZI, Efraim y PADILLA ORTEGA, Rafael. Seminario sobre producción agrícola en Yucatán. Mérida, Yucatán, México: Gobierno del Estado de Yucatán, Secretaría de Programación y Presupuesto, Secretaría de Agricultura y Recursos Hidráulicos, Colegio de Posgraduados, 1980.

HOLLING, Crawford S. Resilience and stability of ecological systems. Annual Review of Ecology and Systematics, v. 4, p.1-23, 1973.

HUTCHINSON, George E. Concluding remarks. Cold Spring Harbor Symposium on Quantitative Biology, v.22, p. 415-27, 1957.

INFANTE RAMÍREZ, Karla D. y ARCE IBARRA, Ana M. Percepción local de los servicios ecológicos y de bienestar de la selva de la zona maya en Quintana Roo, México. Investigaciones Geográficas, Boletín del Instituto de Geografía, UNAM, n. 86, p. 67-81, 2015.

LEOPOLD, Aldo. Game management. New York: Charles Scribner Sons, 1933.

LOTKA, Alfred J. Undamped oscillations derived from the law of mass action. Journal of American Chemical Society, v. 42, p. 1595-1598, 1920.

MARIACA MÉNDEZ, Ramón. La milpa maya yucateca en el siglo XVI: Evidencias etnohistóricas y conjeturas. Etnobiología, v. 13, n. 1, p. 1-25, 2015.

MÉNDEZ, V. Ernesto; BACON, Chris M. \& COHEN Rose. Agroecology as a transdisciplinary, participatory, and action-oriented approach. Agroecology and Sustainable Food Systems, special issue: Agroecology and the transformation of agri-food systems: Transdisciplinary and participatory perspectives v. 37, n. 1, p. 3-18, 2015.

ODUM, Eugene P. Fundamentals of Ecology. 1st ed. Philadelphia: Saunders. (2nd ed., 1959; 3rd ed., 1971), 1953.

OKAGOR, Monica, \& NOAH, Paulinus. The role of local languages in sustainable community development projects in Ebonyi State, Nigeria. European Scientific Journal, v. 1, n. 35, p. 272-283, 2014. 
ORTIZ, Marielsa y BORJAS, Beatriz. La Investigación Acción Participativa: aporte de Fals Borda a la educación popular. Espacio Abierto, v.17, n. 4, p. 615-627, octubrediciembre. Universidad del Zulia, Venezuela, 2008.

REYES-GARCÍA, Victoria. Conocimiento ecológico tradicional para la conservación: dinámicas y conflictos. Papeles, edición especial, n. 107, p. 39-55, 2009.

ROGOFF, Barbara. Developing destinies. A Mayan midwife and town. New York: Oxford University Press, 2011.

ROSADO-MAY, Francisco J. 2014. Conceptos ecológicos clásicos presentes en el conocimiento de los pueblos originarios prehispánicos. Bases para el desarrollo sostenible comunitario. II Congreso de las Universidades Interculturales. Universidad Intercultural del Estado de México. San Felipe del Progreso, Edo. de México, 16 de mayo de 2014.

ROSADO-MAY, Francisco J. The Intercultural Origin of Agroecology: Contributions from Mexico. In V.E. Méndez, C.M. Bacon, R. Cohen and S.R. Gliessman (Eds.) Agroecology: A Transdisciplinary, Participatory and Action-Oriented Approach. Advances in Agroecology Series. Boca Raton: CRC Press/Taylor and Francis. Chapter 8, p.123-138, 2015.

ROSADO-MAY, Francisco J. Gestión académica con la milpa y los milperos. ¿Estamos haciendo un buen trabajo en las universidades? $X$ Congreso Mexicano de Etnobiología, Mérida, Yucatán, México, 23 de septiembre, 2016.

ROSADO-MAY, Francisco J. y CUEVAS ALBARRÁN, Valeria B. El programa educativo "Ingeniería en Desarrollo Empresarial" de la Universidad Intercultural Maya de Quintana Roo. ¿Qué justifica su creación? En: E. Brito Estrella (Coord.) Empresa, sostenibilidad y desarrollo regional. José Ma. Morelos, Quintana Roo, México: Universidad Intercultural Maya de Quintana Roo, p. 43-63, 2015.

ROSADO-MAY, Francisco J. \& KISSMANN, Susanne. Planning for sustainable tourism in the southern coast of the Mexican Caribbean. In: L. Hens, R.J. Borden, S. Suzuki \& G. Caravello (Eds.). Research in Human Ecology: An Interdisciplinary Overview. Brussells, Belgium: VUB University Press, p. 53-77, 1998.

SAYRE, Nathan F. The genesis, history and limits of carrying capacity. Annals of the Association of American Geographers, v. 98, n. 1, p. 120-134, 2008.

SEVILLA GUZMÁN, Eduardo y SOLER MONTIEL, Marta. Del desarrollo rural a la agroecología. Hacia un cambio de paradigma. Documentación Social v. 155, p. 25-41, 2009.

TANSLEY, Arthur G. 1935. The use and abuse of vegetational concepts and terms. Ecology, 16:284-307.

TAUBE, Karl. Ancient and contemporary Maya conceptions about field and forest. In: A: Gómez-Pompa, M.F. Allen, S.L. Feddick, and J.J. Jiménez-Osornio (Eds). The lowland Maya area. Three millennia at the human wildland interface. Binghamton, New York: Food Products Press, p. 461- 492, 2003.

TERAN, Silvia, y RASMUSSEN, Christian. La milpa de los Mayas. Mérida, Yucatán, México: Gobierno del Estado de Yucatán, 1994.

THOMAS, R. K. Cherokee values and worldview. University of North Carolina. Disponible en http://works.bepress.com/robert thomas/40, 1958.

TUZ CHÍ, Lázaro H. Así es nuestro pensamiento. Cosmovisión e identidad en los rituales agrícolas de los Mayas Peninsulares. Tesis Doctoral, Universidad de Salamanca, Instituto de Iberoamérica. Facultad de Ciencias Sociales, p. 365, 2009.

UNITED NATIONS EDUCATIONAL, SCIENTIFIC and CULTURAL ORGANIZATION. Why language matters for the millennium development goals. Bangkok, Thailand, Asia and Pacific Regional Bureau for Education: UNESCO, 2012.

VOLTERRA, Lotka. Lecons sur le theorie mathematique de la lutte pour la vie. Paris: Gauthiers-Villars, 1931. 


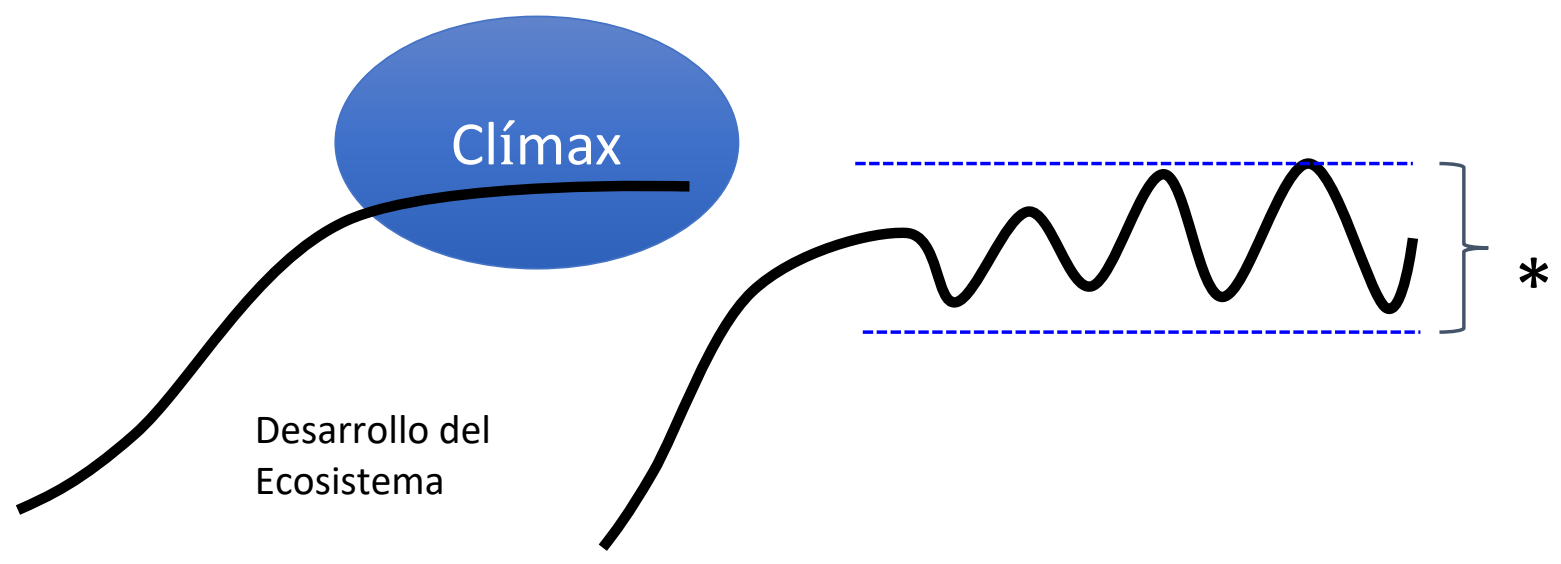

*Ecosistema en su rango de equilibrio dinámico

Figura 1. Modelos de desarrollo de un ecosistema. 